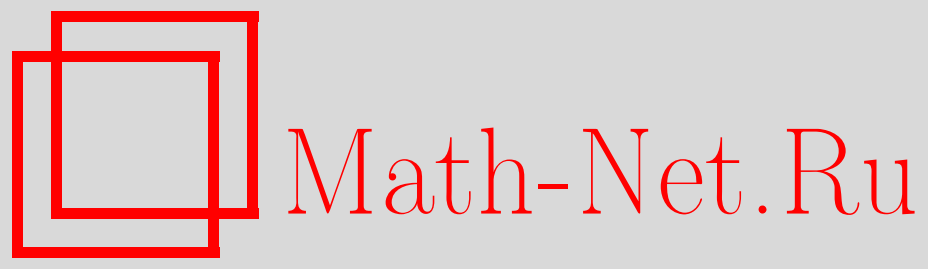

М. В. Ружанский, Поточечная лемма ван дер Корпута для функций нескольких переменных, Функи. анализ и его прил., 2009, том 43, выпуск 1, 91-93

DOI: https://doi.org/10.4213/faa2937

Использование Общероссийского математического портала MathNet.Ru подразумевает, что вы прочитали и согласны с пользовательским соглашением

http://www.mathnet.ru/rus/agreement

Параметры загрузки:

IP: 35.173 .137 .237

26 апреля 2023 г., 13:23:58

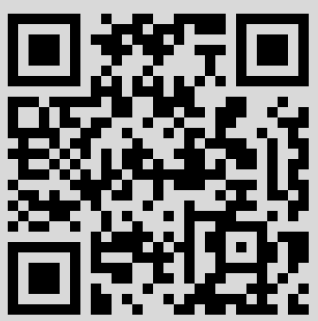


УДК 517

\title{
Поточечная лемма ван дер Корпута для функций нескольких переменных*
}

\author{
(c) 2009. М. В. РужАнСКИЙ
}

Посвящается памяти Л. Р. Волевича

1. Введение. Одномерная лемма ван дер Корпута хорошо известна в гармоническом и асимптотическом анализах для функций одной переменной, где она играет фундаментальную роль в асимптотических оценках осциллирующих интегралов. В настоящей статье мы предложим многомерную формулировку этого результата. В одномерном случае вопрос поведения осциллирующего интеграла

$$
I(\lambda)=\int_{\mathbb{R}} e^{i \lambda \Phi(y)} a(y) d y
$$

при вещественных $\lambda \rightarrow \infty$ хорошо изучен. Известно, что если $a \in C_{0}^{\infty}(\mathbb{R})-$ функция с достаточно малым компактным носителем и $\Phi-$ вещественнозначная гладкая функция, то из условия $\Phi^{(m)}\left(y_{0}\right) \neq 0$ для некоторого $y_{0} \in \operatorname{supp} a$ следует оценка $|I(\lambda)| \leqslant C \lambda^{1 / m}$ для интеграла. Эта оценка называется леммой ван дер Корпута.

2. Формулировка результатов. Оказывается возможным предложить многомерную формулировку леммы ван дер Корпута. Мы также распространим ее на случай комплекснозначных фазовых функций, зависящих от параметра ситуацию, часто возникающую в приложениях.

Теорема 1. Рассмотрим осииллирующий интеграл

$$
I(\lambda, \nu)=\int_{\mathbb{R}^{n}} e^{i \lambda \Phi(y, \nu)} a(y, \nu) \chi(y) d y,
$$

где размерность $n$ больше или равна 1, а - произволъный параметр. Предположим, что $\chi \in C_{0}^{\infty}(\Omega)$ для некоторого ограниченного множества $\Omega \subset \mathbb{R}^{n}$. Пусть $\gamma \geqslant 2$ - такое челое число, что выполнены следуюшие предположения:

1) амплитуда $a(y, \nu)$ удовлетворяет оценке $\left|\partial_{y}^{\alpha} a(y, \nu)\right| \leqslant C_{\alpha}$ для всех мультииндексов $\alpha$ длины $|\alpha| \leqslant[n / \gamma]+1$, всех $y \in \Omega$ и всех параметров $\nu$ (здесъ $[n / \gamma]$ - иелая часть числа $n / \gamma)$;

2) комплекснозначная фаза $\Phi(y, \nu)$ удовлетворяет условию $\operatorname{Im} \Phi(y, \nu) \geqslant 0$ для всех $y \in \Omega$ и всех параметров $\nu, u$, кроме того, для некоторого $y_{0} \in \mathbb{R}^{n}$ фнкиия

$$
F(\rho, \omega, \nu):=\Phi\left(y_{0}+\rho \omega, \nu\right)
$$

удовлетворяет неравенствам

$$
\left|\partial_{\rho} F(\rho, \omega, \nu)\right| \geqslant C \rho^{\gamma-1} \quad u \quad\left|\partial_{\rho}^{m} F(\rho, \omega, \nu)\right| \leqslant C_{m} \rho^{1-m}\left|\partial_{\rho} F(\rho, \omega, \nu)\right|
$$

для всех $\omega \in \mathbb{S}^{n-1}$, всех параметров $\nu$, всех иелых $m$, таких, что $1 \leqslant m \leqslant$ $[n / \gamma]+1, u$ всех $\rho>0$, для которих $y_{0}+\rho \omega \in \Omega$.

*Работа поддержана грантом фонда Leverhulm Trust и грантом EPSRC EP/E062873/1. 
Тогда найдется такая константа $C=C_{n, \gamma}>0$, зависящая от амплитуды и от констант в неравенствах (3), что выполнена оценка

$$
|I(\lambda, \nu)| \leqslant C(1+\lambda)^{-n / \gamma} \quad \text { для всех } \lambda \in[0, \infty) \text { и всех параметров } \nu .
$$

Условие (3) на фазовую функцию $\Phi$ является ключевым условием теоремы. Поэтому возникает естественный вопрос, когда оно выполнено. Оказывается, что если первые $\gamma$ членов ряда Тейлора функции $F$ не обращаются одновременно в нуль, а также выполнено некоторое условие выпуклости, то этого достаточно для выполнения условия (3). Сформулируем это более точно.

Пусть $F=F(\rho, \mu)$ - комплекснозначная функция, гладкая по $\rho \in[0, \infty)$ для всех значений параметра $\mu$ в произвольном пространстве параметров. Разложим функцию $F(\cdot, \mu)$ в ряд Тейлора по $\rho$ в точке $\rho=0$, так что

$$
F(\rho, \mu)=\sum_{j=0}^{N} a_{j}(\mu) \rho^{j}+R_{N}(\rho, \mu),
$$

где $R_{N}(\rho, \mu)$ - остаточный член. Будем говорить что функция $F-$ функция выпуклого типа порядка $\gamma \geqslant 2$, если для некоторого $\delta>0$ выполнены следующие условия:

- $a_{0}(\mu)=a_{1}(\mu)=0$ для всех параметров $\mu$;

- существует такая константа $C>0$, что неравенство

$$
\sum_{j=2}^{\gamma}\left|a_{j}(\mu)\right| \geqslant C
$$

выполнено для всех параметров $\mu$;

- для всех параметров $\mu$ функция $\left|\partial_{\rho} F(\rho, \mu)\right|$ является неубывающей по $\rho$ в промежутке $0<\rho<\delta$;

- для всех $k \in \mathbb{N}$ функция $\partial_{\rho}^{k} F(\rho, \mu)$ равномерно ограничена по $0<\rho<\delta$ и $\mu$.

Такие функции удовлетворяют ключевому условию (3) теоремы 1 :

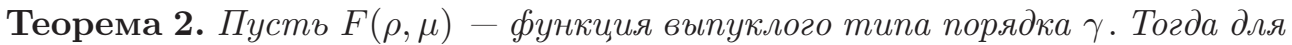
каждого достаточно малого $0<\delta \leqslant 1$ существуют константы $C, C_{m}>0$, maкuе, чmo

$$
\left|\partial_{\rho} F(\rho, \mu)\right| \geqslant C \rho^{\gamma-1} \quad \text { u }\left|\partial_{\rho}^{m} F(\rho, \mu)\right| \leqslant C_{m} \rho^{1-m}\left|\partial_{\rho} F(\rho, \mu)\right|
$$

для всех $0<\rho<\delta$, всех параметров $\mu$ и всех $m \in \mathbb{N}$.

3. Обсуждение. 1. Отметим, что если функция $F$ выпуклого типа вещественнозначна, то, используя равенство $\partial_{\rho} F(0, \mu)=0$, легко получить, что условие неубывания функции $\left|\partial_{\rho} F(\rho, \mu)\right|$ по $\rho$ эквивалентно одному из условий $\partial_{\rho}^{2} F(\rho, \mu) \geqslant 0$ либо $\partial_{\rho}^{2} F(\rho, \mu) \leqslant 0$ для всех $0<\rho<\delta$. Поэтому мы называем такие функции $F$ функциями выпуклого типа.

2. Варианты утверждения теоремы 2 для аналитических функций $F(\rho)$, не зависящих от параметра $\mu$, были известны ранее ([2], [4], [7]). Доказательства использовали оценку остатка ряда Тейлора, основанную на интегральной формуле Коши для аналитических функций. Таким образом, распространение утверждения на гладкие функции требует принципиально другого доказательства. 
Заметим также, что утверждение теоремы 1 отличается от оценки, получаемой методом стационарной фазы, так как стационарная точка, вообще говоря, вырождается с высоким порядком. Кроме того, оценка из теоремы 1 связана с индексом осцилляции фазовой функции $\Phi$ (см. [1]), однако условия, накладываемые нами на фазу осциллирующего интеграла, существенно отличаются от условий из [1].

3. Теорема 1 может быть использована в многочисленных случаях, например, когда требуется оценивать осциллирующие интегралы, в свою очередь являющиеся интегральными ядрами интегральных операторов Фурье. Обычно из них следуют $L^{p}-L^{p^{\prime}}$-оценки для соответствующих интегралов Фурье. В случае $L^{p}-L^{p}$-оценок ситуация более геометрическая, и в работе [5] можно найти обзор этой темы. Объединяя оценки этих типов и интерполируя, можно получить полный набор оценок типа $L^{p}-L^{q}$ для интегральных операторов Фурье соответствующего класса.

4. Возможность зависимости фазы и амплитуды от параметров принципиальная. Так, теорема 1 играет ключевую роль в получении дисперсионных оценок и последующих оценок Стрихарца для гиперболических уравнений и систем. Например, в работе [6] теорема 1 используется для получения дисперсионных оценок для гиперболических уравнений высоких порядков с постоянными коэффициентами. В этом случае, при высоких частотах, члены низших порядков можно рассматривать как возмущение главной части и параметр $\nu$ содержит в себе информацию об этих возмущениях. В то же время в работе [3] теорема 1 используется для получения дисперсионных оценок для гиперболических уравнений с однородными символами, но с коэффициентами, зависящими от времени. В этом случае роль параметра $\nu$ другая, он содержит в себе информацию о поведении коэффициентов уравнения при больших временах.

5. Доказательство теоремы 1 основывается на том, что, благодаря условию (3), фазовая функция обладает определенной невырожденностью по всем радиальным направлениям. Таким образом, задача может быть сведена к соответствующей одномерной оценке, в свою очередь применяемой равномерно по всем направлениям. Отсюда и следует оценка (4), являющаяся $n$-мерным усилением соответствующей одномерной оценки.

\section{ЛитерАтУРА}

[1] В. И. Арнольд, А. Н. Варченко, С. М. Гусейн-Заде, Особенности дифберенцируемых отображений, т. 2, Наука, 1984. [2] R. M. Beals, Mem. Amer. Math. Soc., 38:264 (1982). [3] T. Matsuyama, M. Ruzhansky, Asymptotic integration and dispersion for hyperbolic equations, with applications to Kirchhoff equations, http://arxiv.org/abs/0709.1678v1. [4] B. Randol, Trans. Amer. Math. Soc., 139 (1969), 279-285. [5] М. В. Ружанский, УМН, 55:1 (2000), 99-170. [6] M. Ruzhansky, J. Smith, Dispersive and Strichartz estimates for hyperbolic equations with constant coefficients, http://arxiv.org/abs/0711.2138. [7] M. Sugimoto, Math. Z., 215:4 (1994), $519-531$.

Imperial College London e-mail: m.ruzhansky@imperial.ac.uk 